Research Paper

\title{
Nab-paclitaxel as First Line Treatment for NSCLC in Elderly Patients More Than 75 Years Old
}

\author{
Paul Zarogoulidis ${ }^{\circledR \star *}$, Haidong Huang ${ }^{3^{*}}$, Chong Bai ${ }^{3^{*}}$, Dimitris Petridis ${ }^{4}$, Susana Papadopoulou ${ }^{5}$, Eleni \\ Faniadou', Ellada Eleftheriadou1, Georgia Trakada ${ }^{6}$, Kosmidis Cristoforos ${ }^{7}$, Aggeliki Rapti ${ }^{8}$, Lonny \\ Yarmus $^{9}$, David-Feller Kopman ${ }^{9}$, Yan-Gao Man ${ }^{10}$, Wolfgang Hohenforst-Schmidt2* \\ 1. Pulmonary Oncology Unit, “G. Papanikolaou” General Hospital, Aristotle University of Thessaloniki, Thessaloniki, Greece; \\ 2. Medical Clinic I, "Fuerth" Hospital, University of Erlangen, Fuerth, Germany; \\ 3. Department of Respiratory and Critical Care Medicine, Changhai Hospital, The Second Military Medical University, Shanghai, China; \\ 4. Department of Food Technology, School of Food Technology and Nutrition, Alexander Technological Educational Institute, Thessaloniki, Greece; \\ 5. Department of Nutrition and Dietetics, Alexander Technological Educational Institute, Thessaloniki, Greece; \\ 6. Division of Pulmonology, Department of Clinical Therapeutics, National and Kapodistrian University of Athens School of Medicine, Alexandra Hospital, \\ Athens, Greece; \\ 7. General Surgery Department, European Interbalkan Medical Center, Thessaloniki, Greece; \\ 8. Second Pulmonary Clinic, 'Sotiria' Chest Diseases Hospital, Athens, Greece; \\ 9. Division of Pulmonary and Critical Care Medicine, Sheikh Zayed Cardiovascular \& Critical Care Tower, Baltimore, U.S.A; \\ 10. Research Laboratory and International Collaboration, Bon Secours Cancer Institute, VA, USA. \\ * Paul Zarogoulidis, Wolfgang Hohenforst-Schmidt, Haidong Huang and Chong Bai contributed equally to this work.
}

$\triangle$ Corresponding author: Paul Zarogoulidis, M.D, Ph. D, Pulmonary Department-Oncology Unit, "G. Papanikolaou" General Hospital, Aristotle University of Thessaloniki, Thessaloniki, Greece Fax: 00302310992424 Mobile: 00306977271974 E-mail: pzarog@hotmail.com

(C) Ivyspring International Publisher. This is an open access article distributed under the terms of the Creative Commons Attribution (CC BY-NC) license (https://creativecommons.org/licenses/by-nc/4.0/). See http://ivyspring.com/terms for full terms and conditions.

Received: 2017.02.03; Accepted: 2017.05.03; Published: 2017.06.04

\begin{abstract}
Introduction: Lung cancer is still the leading cause of cancer among cancer patients. Although there are novel therapies as second line treatment for NSCLC, there is an issue for elderly patients. Patients and Methods: We collected retrospectively data from 60 patients $>75$ years of age. Thirty of these patients received nab-paclitaxel and first line treatment and were compared to thirty patients that received only best supportive care. Results: The median life of patients at the date of disease progression, although increased by the administration of the drug $(92$ days versus 70 ) was not confirmed statistically significantly (Mann-Whitney test: $W=280, p=0.138$ ). The administration of drug seems to keep stable the biological condition of patients (McNemar's test: $X^{2}=0.033, p=0.99$ ). Patients with chemotherapy the death rate was increased by $50 \%$ as compared to those with best supportive care (12 vs 8$)$, the median life until the unfortunate event surpassed statistically significantly the latter (150 days of life as compared to 108, Mann-Whitney test: $W=57.5, p=0.045$ ). Discussion: Nab-paclitaxel as a monotherapy could be considered as a first line treatment option for patients $>75$ years of age without any previous cardiological medical history when compared to best supportive care.
\end{abstract}

Key words: NSCLC, nab-paclitaxel, Abraxane, adenocarcinoma, squamus, chemotherapy.

\section{Introduction}

Lung cancer still remains the leading cause of death among cancer patients with an increasing rate among women. [1] Lung cancer is still underdiagnosed due to the lack of early disease symptoms and blood markers. A hallmark in late stage disease for non-small cell lung cancer was the discovery of targeted therapy with tyrosine kinase inhibitors (TKIS) for adenocarcinoma as first line treatment.[2] Later on immunotherapy followed as second line treatment for adenocarcinoma and squamous cell carcinoma.[3] It is known that non-specific cytotoxic agents induce adverse effects and therefore it is of benefit for the patient if possible to receive a treatment without adverse effects or less 
than those observed with chemotherapy.[4] Recently afatinib was approved as second line treatment for squamous cell carcinoma, providing to treating physicians an additional less toxic therapy.[5] Currently an issue that treating physicians have to cope with is the group of the elderly patients more than 75 years of age which are newly diagnosed and cannot receive targeted therapy. Although we have several treatment choices for second line treatment we do not have many choices for first line treatment. Regarding which patient is considered elder, we have observed through the published literature that for Europe the elderly are considered those $>75$, but for US $>65$ years of age. $[6,7]$ Performance status is very important and in most cases the choice of treatment for the elderly is based on this. There are data regarding second line treatment with nab-paclitaxel as second line treatment for the elderly, however; there are no data regarding whether it could be used as first line treatment.[8] Moreover; there are recently published data regarding the efficacy of nab-paclitaxel in combination with carboplatin for non-small cell lung cancer.[9] Nab-paclitaxel belongs to the taxane family, however; it is designed in a so that it has a local sustain release effect and deep penetration in tumor tissue. Moreover; it is faster diffused within cancer tissue than normal tissue.[10] Regarding the elderly patients there are still no data regarding nab-paclitaxel as first line treatment and this is what we investigated in our retrospective study.

\section{Patients and Methods}

All patients met the following inclusion criteria: 1) they had a histologic or cytologic diagnosis of stage IV NSCLC, 2) they were $\geq 75$ years, 3) they had an Eastern Cooperative Oncology Group PS \#2, 4) they had not received our first-line chemotherapy or targeted therapy, 5) no known heart disease and ejection fraction $\geq 40 \%$ and 6 ) they had measurable disease as defined by Response Evaluation Criteria in Solid Tumors (RECIST).[11] Patients were excluded if they had symptomatic brain metastases or preexisting peripheral neuropathy (grade 1 or higher) and symptomatic heart disease. We could not evaluate elderly patients by comprehensive geriatric assessment due to some patients with unknown sociodemographic factors. Our study was approved by the investigational review board (IRB) of our hospital "G. Papanikolaou" University General Hospital, as a retrospective study. Informed consent for every patient is always acquired upon initiation of the patient therapy.
Nab-paclitaxel was administered weekly on days 1,8 , and 15, followed by 2 weeks of rest. This mode of administration has been previously used.[12] Treatment was repeated every 5 weeks until disease progression or unacceptable toxicity. A dose of 100 $\mathrm{mg} / \mathrm{m}^{2}$ was administered for $\sim 30$ minutes with corticosteroid and antihistamine premedication as per our hospitals protocol. The protocol recommended that if patients experienced grade 3 or 4 neutropenia or thrombocytopenia during treatment, they should receive a subcutaneous injection of granulocyte colony-stimulating factor or epoetin alfa to address such hematological toxicities.

The primary efficacy end point was overall response rate (ORR), defined as the percentage of patients having either a complete response (CR) or a partial response (PR), determined according to the RECIST guidelines.[11] Secondary efficacy end points included disease control rate (DCR; defined as the percentage of patients with stable disease [SD] for 16 weeks and $\mathrm{CR}$ and $\mathrm{PR}$ ), progression-free survival (PFS; defined as the time from treatment to the objective progression of disease), and overall survival (OS; defined as the time from treatment to death). Response assessments were performed every 5 weeks with a chest $\mathrm{x}$-ray and every 5 weeks according to RECIST. All patients had a baseline computed tomography (CT) examination of the chest and a reassessment every 4 treatment cycles. Hematologic (every week after each treatment) and imaging examinations (every 5 weeks with chest $x$-ray) were routinely performed during treatment with nab-paclitaxel. The safety end points were as follows: 1) the incidence of treatment-related AEs based on National Cancer Institute Common Toxicity Criteria Version 2.0, 2) laboratory abnormalities, and 3) serious AEs.

All analyses were performed using statistical software.

\section{Results}

We retrospectively reviewed 60 patients with Stage IV, 30 who received nab-paclitaxel as first-line treatment and 30 without (best supportive care) from March 2016 to January 2017 in the "G. Papanikolaou" General Hospital, Thessaloniki, Greece.

The median life of patients at the date of disease progression, although increased by the administration of the drug (92 days versus 70) was not confirmed statistically significantly (Mann-Whitney test: $\mathrm{W}=$ $280, p=0.138$ ). Figure 1 shows the distribution of patients from both treatments as they affected time of progression and survival time. 


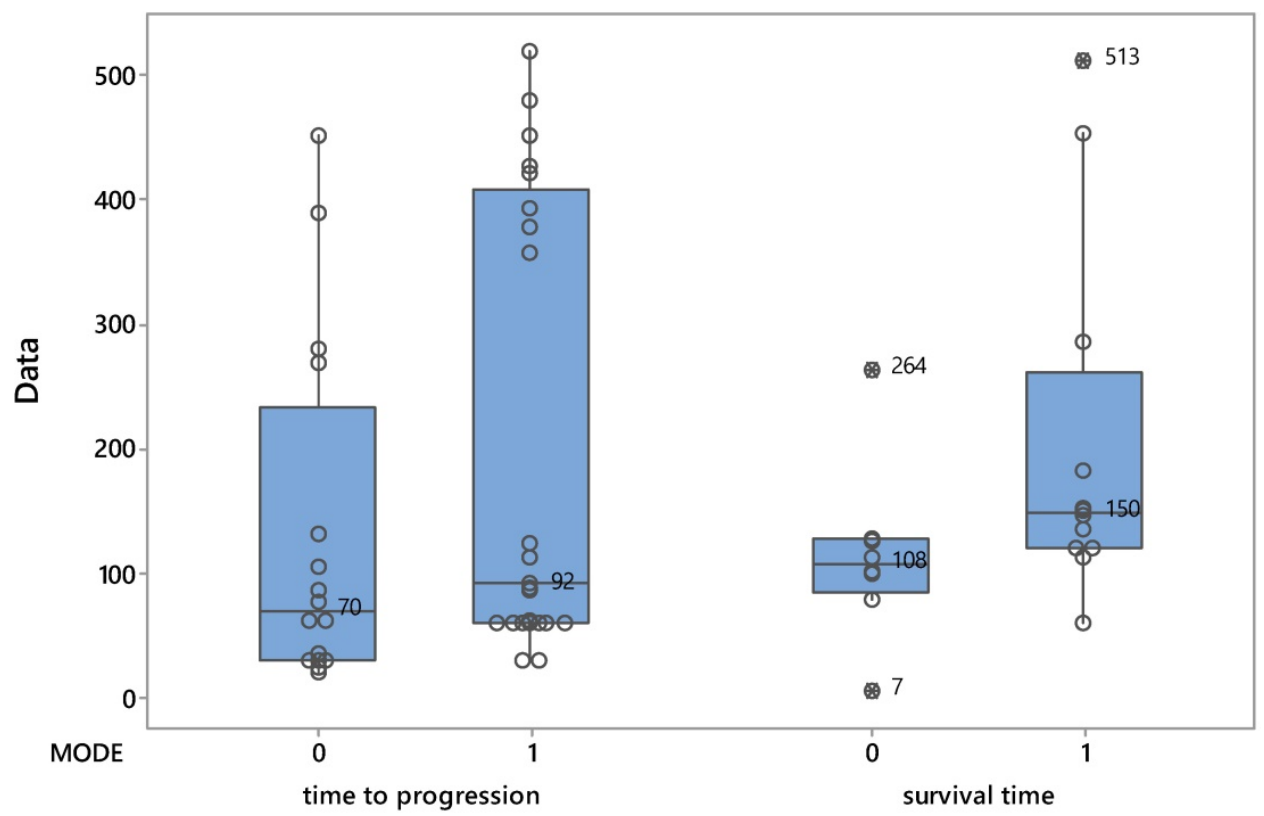

Figure 1. Boxplots describing the evolution of patients based on the two protocols.
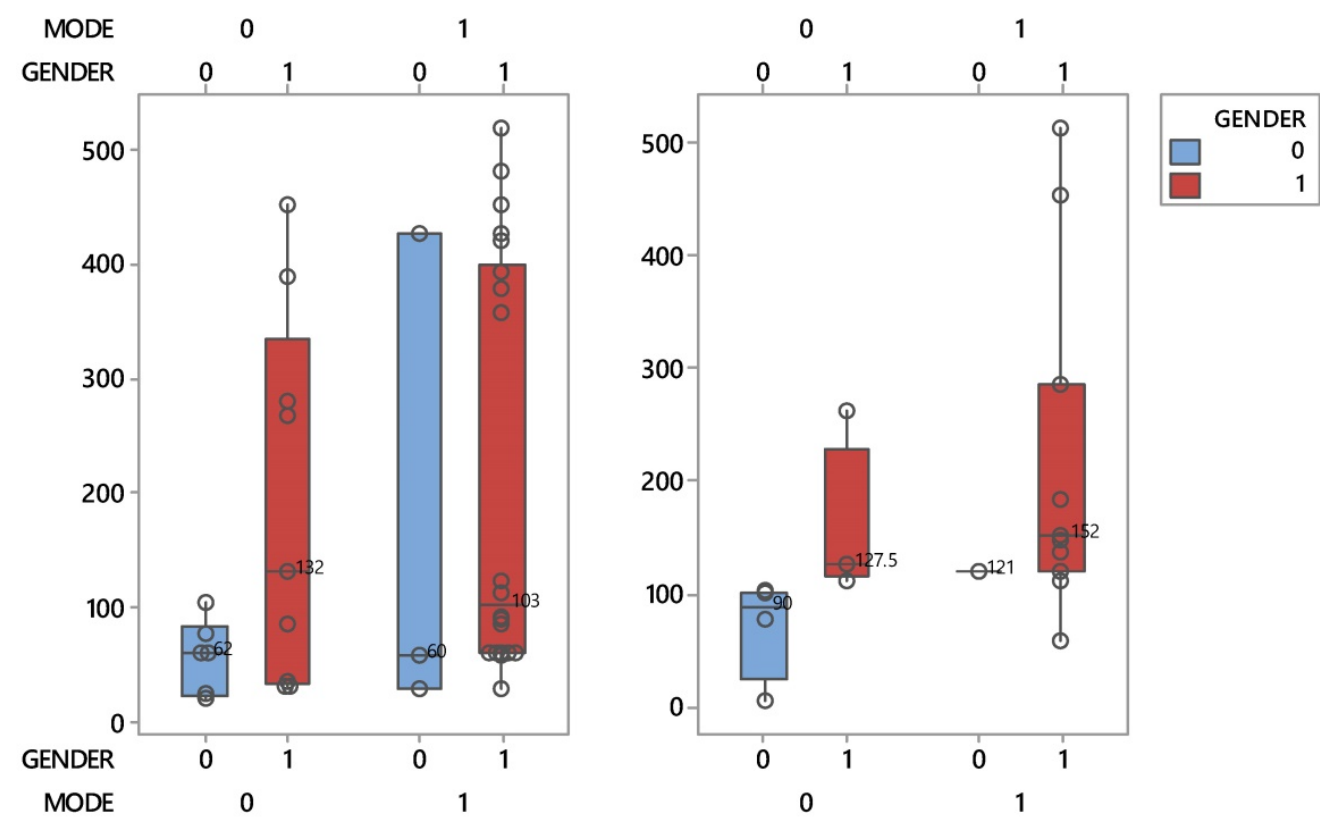

Figure 2. Boxplots describing the interactive effects between patient protocols and sex during the course of the disease process (left) and life (right).

In contrast, although in patients with chemotherapy the death rate was increased by $50 \%$ as compared to those with best supportive care (12 vs 8$)$, the median life until the unfortunate event surpassed statistically significantly the latter (150 days of life as compared to 108, Mann-Whitney test: $\mathrm{W}=57.5, \mathrm{p}=$ $0.045)$.

It is worthy to mention that women patients belonging to the conventional group, recorded a shorter lifespan than mean. Gender seems to play an important role in the life of patients and only in the supportive care group. Indeed, this group in Figure 2 is distinguished by a clear reduction of the median survival time of women (50 days), 2.5 times lower than that of men (127.5 days). It is reminded, that two medians whose intequartiles (boxes) do not overlap, they differ statistically significantly. This striking effect does not occur when disease progress matters.

The administration of drug seems to keep stable the biological condition of patients or at least that does not worsen (McNemar's test: $\chi^{2}=0.033, p=0.99$, Table 1). In fact, as shown by the cross-tabulated frequencies of both performance statuses PS (upon initiation and follow up), the patients have not 
deteriorated after using the drug. 12 patients remained steadily in good condition before and after (00), 7 aggravated their health, 8 improved their profile from 1 to 0 , while 3 patients did not switch from bad condition [11].

Table 1. Cross-tabulation of patients submitted to chemotherapy showing particular performance status upon initiation (PSI) and upon followup (PS2). $0=$ good condition and $1=$ moderate to bad condition

\begin{tabular}{llll}
\hline PS1 & PS2 & \multicolumn{2}{c}{ All } \\
\cline { 2 - 3 } & 0 & 1 & \\
\hline 0 & 12 & 7 & 19 \\
1 & 8 & 3 & 11 \\
All & 20 & 10 & 30 \\
\hline
\end{tabular}

The condition of patients in the conventional group showed a significant deterioration (McNemar's test: $\left.\chi^{2}=4.5, \mathrm{p}=0.034\right)$. According to their performance status (Table 2, cross-tabulated frequencies), of the 30 patients, 4 remained in good health until disease progression (00), 14 showed signs of deterioration (01), 4 improved their condition (10) and 8 did not change from bad condition (11).

Table 2. Cross-tabulation of patients in the conventional group showing particular performance status upon initiation (PS1) and upon followup (PS2). $0=$ good condition and $1=$ moderate to bad condition

\begin{tabular}{llll}
\hline PS1 & \multicolumn{2}{l}{ PS2 } & All \\
\cline { 2 - 3 } & 0 & 1 & 18 \\
\hline 0 & 4 & 14 & 12 \\
1 & 4 & 8 & 30 \\
All & 8 & 22 & \\
\hline
\end{tabular}

Another notable difference between the two protocols is found in the metastasis detection points after inspecting the disease progression. Figure 3 easily reveals that lung metastases prevail exclusively in patients with chemotherapy, spotted in 11 out of 25 $(44 \%)$ and totaling up to $27.5 \%$ of 40 investigated patients. On the other hand the incidence of metastasis in brain (2) and bones (3) is nearly a unique event in patients treated with the conventional protocol. Tables 3-4.

Table 3. Baseline Characteristics

\begin{tabular}{lll}
\hline Clinical features & Nab-Paclitaxel Group & Best Supportive Care \\
\hline Sex & & \\
Male & 27 & 18 \\
Female & 3 & 12 \\
Age & 77.63 & 77.76 \\
PS 0 upon diagnosis & 19 & 6 \\
PS 1 upon diagnosis & 11 & 24 \\
PS 0 upon follow up & 18 & 23 \\
PS 1 upon follow up & 12 & 7 \\
\hline
\end{tabular}

Table 4. Adverse effects

\begin{tabular}{lll}
\hline & Nab-Paclitaxel Group & Best Supportive Care \\
\hline Cardiac & 3 & 6 \\
Dysphagia & 1 & 9 \\
Neutropenia & 5 & 0 \\
Thrombocytopenia & 9 & 0 \\
Alopecia & 21 & 0 \\
Fatigue & 15 & 9 \\
Hypersensitivity & 1 & 0 \\
*Regarding neutropenia and thrombocytopenia the numbers reflect the number of \\
patients that presented these adverse effects and not the number of subcutaneous \\
injections
\end{tabular}

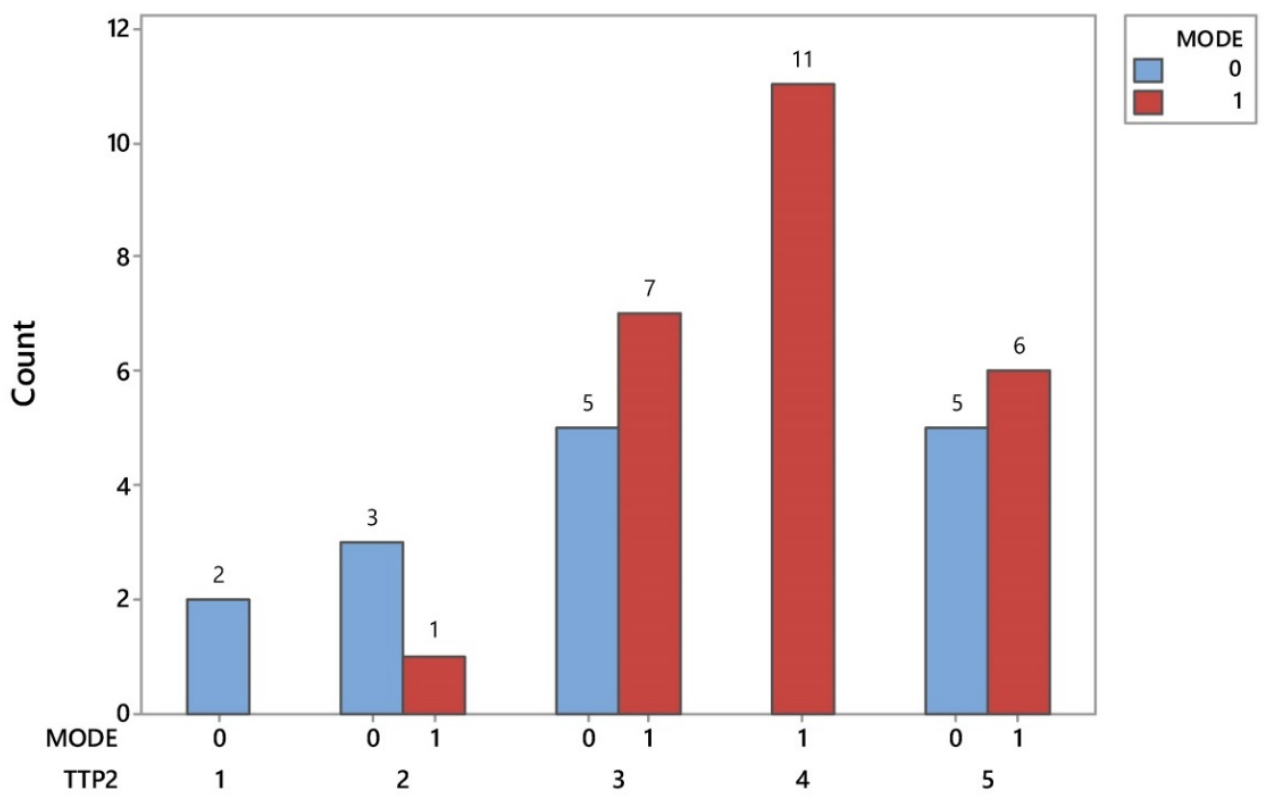

Figure 3. Distribution of identifying signs of metastases in patients of both protocols. TTP2= time to progression; 1) Brain, 2) Bone, 3)Belly, 4) Chest, 5) Combination 


\section{Discussion}

Nab-paclitaxel has been used for several cancers [12] such as; breast cancer and pancreatic cancer [13, 14]. Moreover; in a recent publication by Solimann HH. et al. [15] it is suggested that nab-paclitaxel could enhance the efficacy of checkpoint inhibitors such as; immunotherapeutic agents. In another study by Li Z . et al. [16] it was observed that increased expression of Secreted Protein Acidic and Rich in Cysteine (SPARC) in gastric cancer was a prognostic factor for worse treatment result. The same observation was made for rectal cancer [17]. In the study by Kurtul N. et al. [18] increased SPARC expression was associated with poor prognosis when patients were treated with nab-paclitaxel and concurrent radiotherapy. In the study by Kaira K. et al. [19] a combination of carboplatin and nab-paclitaxel plus radiotherapy was administered as first line in unresectable stage III NSCLC with safety and efficacy in patients $\leq 65$ years of age. Nab-paclitaxel can be used in NSCLC as first line treatment as an alternative to best supportive care in the group of the "elderly" $\geq 75$ years of age. In our study the number of patients is small (60) and the number of adenocarcinoma patients is even smaller (only 12/30NSCLC) in order to observe a clear difference of survival benefit between squamous cell carcinoma and adenocarcinoma (most common diagnosis). Certainly there is a survival benefit from the monotherapy administered. Treating physicians should always considered not only the adverse effects that will follow from a chemotherapy treatment but, also the performance status that will deteriorate when a patient is left without any primary treatment. A balance between therapy and adverse effects is necessary. The cardiac adverse effects in 3 patients receiving nab-paclitaxel occurred within 24 hours of administration and they were considered acute and since the patients were hospitalized they were treated immediately. These patients underwent coronary angiography and where necessary they were treated according to their indication by the cardiologists of our institution. Prior to the medication administration they did not have symptoms/signs of coronary disease as described in the patients and methods section they fulfilled the criteria for inclusion in our study. Our main issues observed in the best support care group was pleural effusion which could not always be dealt with pleurodesis and multiple hospitilisations due to malnutrition and respiratory distress. On the other hand we had two deaths which were attributed to nab-paclitaxel, however; these two patients did not have a previous coronary vessel evaluation. Heart ultrasound which was indeed requested by each patient before chemotherapy evaluation is not enough for this group of patients (elderly). Balancing this information we should check the number of patients that had adverse effects in the best supportive group because they did not receive any therapy. Pericarditis, pleural effusion, malnutrition from enlarged lymph nodes (number 7) and heart tamponade that occurred in these patients, because they did not receive any primary treatment. Jejunostomy which could be applied, is not always acceptable by the patient and there are cases which where the performance status of the patient deteriorates also from the application of this mode of nutrition. Furthermore; hematologic (every week after each treatment) evaluation is indeed necessary for every patient and moreover; the hematologic adverse effects were not as severe as expected. More clinical studies are needed in order to establish chemotherapy monotherapy with nab-paclitaxel for the elderly $\geq 75$ years of age, or probably to a subgroup of these patients. Current data indicate that it can be used when compared to best support care.

\section{Acknowledgments}

This study was supported by Project of Shanghai Municipal Health Bureau (Grant number: 20124166). Project of New Clinical Technology Development of Changhai Hospital (Grant number: NT201506).

\section{Competing Interests}

The authors have declared that no competing interest exists.

\section{References}

1. Siegel RL, Miller KD, Jemal A. Cancer Statistics, 2017. CA: a cancer journal for clinicians. 2017. doi:10.3322/caac.21387.

2. Domvri $K$, Zarogoulidis $\mathrm{P}$, Darwiche $\mathrm{K}$, Browning RF, Li Q Turner JF, et al. Molecular Targeted Drugs and Biomarkers in NSCLC, the Evolving Role of Individualized Therapy. Journal of Cancer. 2013; 4: 736-54. doi: $10.7150 /$ jca.7734.

3. Cortinovis DL, Canova S, Abbate M, Colonese F, Bidoli P. Focus on Nivolumab in NSCLC. Frontiers in medicine. 2016; 3: 67. doi:10.3389/fmed.2016.00067.

4. Hong C, Mei T, Wang J. [Intercalated Combination of Chemotherapy and EGFR-TKIs versus Chemotherapy Alone in the First-line Treatment of Advanced Non-small Cell Lung Cancer: A Meta-analysis]. Zhongguo fei ai za zhi $=$ Chinese journal of lung cancer. 2016; 19: 837-46. doi:10.3779/j.issn.1009-3419.2016.12.06.

5. Keating GM. Afatinib: A Review in Advanced Non-Small Cell Lung Cancer. Targeted oncology. 2016; 11: 825-35. doi:10.1007/s11523-016-0465-2.

6. Saint-Jean $\mathrm{O}$, LeGuen J. [Geriatric intervention in oncology for elderly patients]. Cancer radiotherapie : journal de la Societe francaise de radiotherapie oncologique. 2015; 19: 377-81. doi:10.1016/j.canrad.2015.07.017.

7. Hohenforst-Schmidt W, Zarogoulidis P, Steinheimer M, Benhassen N, Tsiouda T, Baka S, et al. Tyrosine Kinase Inhibitors for the Elderly. Journal of Cancer. 2016; 7: 687-93. doi:10.7150/jca.14819.

8. Jin F, Zhu H, Shi F, Kong L, Yu J. A retrospective analysis of safety and efficacy of weekly nab-paclitaxel as second-line chemotherapy in elderly patients with advanced squamous non-small-cell lung carcinoma. Clinical interventions in aging. 2016; 11: 167-73. doi:10.2147/CIA.S97363.

9. Langer CJ, Hirsh V, Ko A, Renschler MF, Socinski MA. Weekly nab-paclitaxel in combination with carboplatin as first-line therapy in patients with advanced non-small-cell lung cancer: analysis of safety and efficacy in patients with renal impairment. Clinical lung cancer. 2015; 16: 112-20. doi:10.1016/j.cllc.2014.09.003.

10. [Internet] FDA. ABRAXANE® for Injectable Suspension (paclitaxel protein-bound particles for injectable suspension) (albumin-bound). 
https://www.accessdata.fda.gov/drugsatfda_docs/label/2013/021660s037lb 1.pdf

11. Therasse P, Arbuck SG, Eisenhauer EA, Wanders J, Kaplan RS, Rubinstein L, et al. New guidelines to evaluate the response to treatment in solid tumors. European Organization for Research and Treatment of Cancer, National Cancer Institute of the United States, National Cancer Institute of Canada. Journal of the National Cancer Institute. 2000; 92: 205-16.

12. Miyauchi E, Inoue A, Usui K, Sugawara S, Maemondo M, Saito H, Fujita $Y$, Kato T, Suzuki T, Harada T, Watanabe H, Nakagawa T, Ichinose M. Phase II Study of Modified Carboplatin Plus Weekly Nab-Paclitaxel in Elderly Patients with Non-Small Cell Lung Cancer: North Japan Lung Cancer Study Group Trial 1301. Oncologist 2017; doi: 10.1634/theoncologist.2017-0059.

13. Zong $\mathrm{Y}, \mathrm{Wu}$ J, Shen $\mathrm{K}$. Nanoparticle albumin-bound paclitaxel as neoadjuvant chemotherapy of breast cancer: a systematic review and meta-analysis. Oncotarget. 2017. doi:10.18632/oncotarget.14477.

14. Ghosn M, Ibrahim T, Assi T, El Rassy E, Kourie HR, Kattan J. Dilemma of first line regimens in metastatic pancreatic adenocarcinoma. World journal of gastroenterology. 2016; 22: 10124-30. doi:10.3748/wjg.v22.i46.10124.

15. Soliman HH. nab-Paclitaxel as a potential partner with checkpoint inhibitors in solid tumors. OncoTargets and therapy. 2017; 10: 101-12. doi:10.2147/OTT.S122974.

16. Li Z, Li AD, Xu L, Bai DW, Hou KZ, Zheng HC, et al. SPARC expression in gastric cancer predicts poor prognosis: Results from a clinical cohort, pooled analysis and GSEA assay. Oncotarget. 2016; 7: 70211-22. doi:10.18632/oncotarget.12191.

17. Kurtul N, Tasdemir EA, Unal D, Izmirli M, Eroglu C. SPARC: As a prognostic biomarker in rectal cancer patients treated with chemo-radiotherapy. Cancer biomarkers : section A of Disease markers. 2016. doi:10.3233/CBM-161733.

18. Kurtul N, Eroglu C, Unal D, Tasdemir EA, Orhan O, Zararsiz G, et al. Prognostic value of SPARC expression in unresectable NSCLC treated with concurrent chemoradiotherapy. Asian Pacific journal of cancer prevention : APJCP. 2014; 15: 8911-6.

19. Kaira K, Tomizawa $Y$, Imai $H$, Sakurai $R$, Matsuura M, Yoshii A, et al. Phase I study of nab-paclitaxel plus carboplatin and concurrent thoracic radiotherapy in patients with locally advanced non-small cell lung cancer. Cancer chemotherapy and pharmacology. 2016. doi:10.1007/s00280-016-3217-1. 